\title{
Upgrading of Farmed Salmon Oil Through Lipase-Catalyzed Hydrolysis
}

\author{
Derya Kahveci, Mia Falkeborg, Sandra Gregersen and Xuebing Xu*
}

Department of Molecular Biology, Aarhus University, Gustav Wieds Vej 10, 8000 Aarhus C, Denmark

\begin{abstract}
Upgrading of farmed salmon oil obtained from by-products was carried out by lipase-catalyzed hydrolysis to increase omega 3 polyunsaturated fatty acids (PUFA) content. The lipases tested were from Penicillium camembertii (PC), Rhizomucor javanicus (RJ), Rhizopus niveus (RN), Rhizopus delemar (RD), Burkholderia cepacia (BC), Rhizopus oryzae (RO), Candida rugosa (CR) and Rhizomucor miehei (RM). The lipases from PC, RJ and RN had lower hydrolysis degrees (HDs) compared to the rest of the lipases. The lipase from CR had the highest HD after $24 \mathrm{~h}$ (91.89\%). Moreover, CR lipase was the most effective one in concentrating omega 3 PUFA. The final value was increased from $13.77 \%$ to $27.81 \%$ (wt $\%$ ). The changes in omega 3 PUFA content were significantly different among the lipases although the HD values were similar at the end of the reactions, which was believed to be caused by the substrate specificities of the lipases. The investigation of the relationship between HD and hydrolysis resistant value (HRV) for eicosapentaenoic acid (EPA, 20:5), docosahexaenoic acid (DHA, 22:6) and oleic acid (OA, 18:1) revealed that the fatty acid (FA) selectivity of the lipases were significantly different. CR lipase had the highest preference for hydrolyzing OA selectively over EPA and DHA. The reaction conditions, i.e. presence of surfactants, sonication, buffer-to-oil ratio, enzyme load, did not affect the selectivity. Investigation of the reaction conditions revealed that it was possible to obtain $\sim 2.15$-fold of the original omega 3 PUFA content by hydrolysis of salmon oil in the presence of CR lipase (4\%, based on oil weight) with a bufferto-oil ratio of $2: 1(\mathrm{v} / \mathrm{v})$ at $37^{\circ} \mathrm{C}$ for $4 \mathrm{~h}$.
\end{abstract}

Keywords: Candida rugosa lipase, farmed salmon oil, fatty acid selectivity, hydrolysis, hydrolysis resistant value, omega 3 PUFA.

\section{INTRODUCTION}

Omega 3 polyunsaturated fatty acids (PUFA) have been on focus in the past three decades due to their beneficial effects on human nutrition and disease prevention. Eicosapentaenoic acid (EPA, 20:5) and docosahexaenoic acid (DHA, 22:6) are the two most important omega 3 PUFA with welldocumented effects, which have been reviewed recently [1]. The main source of omega 3 PUFA is marine products. EPA and DHA are synthesized by phytoplankton and algae, and are eventually transferred through the food web and are incorporated into lipids of fish and marine mammals [2]. In order to benefit from omega 3 PUFA, fish oil supplements are suggested instead of fish meal consumption.

Concentrated form provides higher omega 3 PUFA content while reducing the intake of saturated and monounsaturated fatty acids (SFA and MUFA, respectively), as well as the total fat intake. For farmed fish, the omega 3 content is usually reduced due to feed containing mainly vegetable oils where a limited amount of long chain PUFA is available. The fish oil value is, therefore, reduced for food uses. This brings huge concern for the fish farming industry, despite the fact that the fish oil quality is considered to be high for food uses due to controlled pollution and better processing operations. Improvement of the omega 3 PUFA content to a high level, at least no less than wild fish oils, is highly demanded, especially by simple, mild and cost efficient processing.

*Address correspondence to this author at the Department of Molecular Biology, Aarhus University, Gustav Wieds Vej 10, 8000 Aarhus C, Denmark; Tel: +45-89425089; Fax: +45-86123178; E-mail: xu@mb.au.dk
Main methods for concentration of omega 3 PUFA are chromatographic separation, fractional or molecular distillation, low-temperature crystallization, supercritical fluid extraction and urea complexation. Lipase-catalyzed hydrolysis has a number of advantages over the abovementioned methods. This process does not involve extremes of $\mathrm{pH}$ and high temperature, which may partially destroy the natural all cis structure of omega 3 PUFA by oxidation, cis-trans isomerization or double bond migration. Mild conditions used reduce the process cost as well. One specific feature of lipasecatalyzed hydrolysis reaction is that, the process results in selective production due to substrate and positional specificities of lipases. Moreover, recovery of omega 3 PUFA in glyceride form is nutritionally more favorable than methyl or ethyl esters of these fatty acids (FAs) because of improved absorption of glyceride form [3]. There have been many attempts to employ lipase-catalyzed hydrolysis to produce omega 3 PUFA concentrates. Several studies showed that the efficiency of concentration depended on the substrate specificity, both acyl chain and molecule specificities, of the lipase used [4-12].

The present study aimed to select a suitable and also practical candidate lipase to concentrate omega 3 PUFA in the farmed salmon oil. Due to low content of omega 3 PUFA in the salmon oil, the discrimination power of the lipases towards omega 3 PUFA is particularly demanding. The concentration efficiency was shown to be dependent on the FA specificity of lipases. Effects of the reaction conditions on the concentration level were investigated as well. 


\section{MATERIALS AND METHODS}

\section{Materials}

Salmon oil from by-products of farmed salmon was provided by Marine Bioproducts A/S (Bergen, Norway) according to the patented enzymatic process [13] involving hydrolysis of by-products by a protease enzyme. Nonimmobilized lipases from Penicillium camembertii (PC; $244.9 \mathrm{U} / \mathrm{g}$ ), Rhizomucor javanicus (RJ; $751.4 \mathrm{U} / \mathrm{g}$ ), Rhizopus niveus (RN; $589.5 \mathrm{U} / \mathrm{g})$, Rhizopus delemar (RD; 1092.5 U/g), Burkholderia cepacia (BC; previously known as Pseudomonas cepacia; $705.1 \mathrm{U} / \mathrm{g}$ ), and Rhizopus oryzae (RO; 914.7 U/g) were from Amano (Virginia, VA, USA) while Candida rugosa lipase (CR; previously known as Candida cylindracea $; 1489.2 \mathrm{U} / \mathrm{g}$ ) was purchased from Fluka (Buchs, Switzerland). Immobilized lipase from Rhizomucor miehei $(\mathrm{RM} ; 282 \mathrm{U} / \mathrm{g}$ ) was provided by Novozymes A/S (Bagsvaerd, Denmark). Fatty acid methyl ester standard was purchased from Nu-Chek-Prep (Elysian, MN, USA). All other reagents and solvents used were from Sigma-Aldrich Co. (St. Louis, MO, USA) and of chromatographic grade.

\section{Methods}

\section{Determination of Enzyme Activities}

Enzyme activities were determined by the Japanese Industrial Standard method [14] substituting salmon oil for olive oil. Free fatty acids (FFA) released by the hydrolysis reaction $(30 \mathrm{~min})$ were titrated against $0.5 \mathrm{~N} \mathrm{NaOH}$ in the presence of $1 \%$ methanolic phenolphthalein solution. One unit of enzyme activity (U) was defined as the amount of enzyme that liberated $1 \mu \mathrm{mol}$ of FA per min at $37^{\circ} \mathrm{C}$.

\section{Screening of Lipases for Lipase-Catalyzed Hydrolysis of Salmon Oil}

Three grams of salmon oil and $6 \mathrm{~mL}$ of $150 \mathrm{mM}$ phosphate buffer ( $\mathrm{pH}$ 7.0) were placed in a $15 \mathrm{~mL}$ vial and sonicated for $30 \mathrm{~min}$. The substrate mixture was then transferred to a sealed jacketed glass reactor of $25 \mathrm{~mL}$ volume and heated to $37^{\circ} \mathrm{C}$ under $700 \mathrm{rpm}$ stirring. After the substrates reached the reaction temperature, lipase $(40 \mathrm{U} / \mathrm{g}$ oil $)$ was added to initiate the reaction. Samples were taken periodically for analysis. $20 \mu \mathrm{L}$ sample was dissolved in $800 \mu \mathrm{L}$ chloroform: methanol $(2: 1, \mathrm{v} / \mathrm{v})$ and analyzed by thin layer chromatography coupled with a flame ionization detector (TLC-FID) to quantify the lipid classes (triacylglycerols, TAG; diacylglycerols, DAG; monoacylglycerols, MAG; FFA). $100 \mu \mathrm{L}$ of sample was mixed with $1 \mathrm{~mL}$ of ethanol and $1 \mathrm{~mL}$ of distilled water. Glyceride fraction was extracted twice by $2 \mathrm{~mL}$ hexane after saponification of FFA by adding $0.5 \mathrm{~mL}$ of $0.5 \mathrm{M}$ ethanolic $\mathrm{KOH}$. The ethanolic water phase was acidified by $0.3 \mathrm{~mL}$ of $2 \mathrm{M} \mathrm{HCl}$, and FFA fraction was extracted by hexane similarly. TLC-FID analysis confirmed that all FFA was reserved in FFA fraction while TAG, DAG and MAG were combined in the glyceride fraction. Fractions were methylated according to the AOCS method Ce-1b 89 [15] and analyzed by gas chromatography (GC).

\section{Effect of Reaction Conditions: Buffer-to-Oil Ratio, Emulsi- fication, Enzyme Load}

To investigate the effects of reaction conditions on omega 3 PUFA concentration, a set of experiments were conducted by CR lipase. The reaction conditions are summarized in Table 1. Products were analyzed similarly.

\section{Lipid Class Analysis by TLC-FID}

Samples were analyzed by thin layer chromatography coupled with a flame ionization detector (Iatroscan MK-6 s, Bechenheim, Germany). Aliquots of $20 \mu \mathrm{L}$ were dissolved in $0.8 \mathrm{~mL}$ of chloroform/methanol mixture $(2: 1, \mathrm{v} / \mathrm{v})$, and $1 \mu \mathrm{L}$ of diluted sample was spotted onto silica-coated Chromarod quartz rods by a semiautomatic sample spotter. Samples were developed with the developing system of $n$-hexane, diethyl ether, and acetic acid $(45: 25: 1, \mathrm{v} / \mathrm{v} / \mathrm{v})$. The rods were dried for $2 \mathrm{~min}$ at $120^{\circ} \mathrm{C}$ prior to analysis. The area percentages of TAG, DAG, MAG, and FFA were used for the calculation of product yields. Hydrolysis degree is defined as (100-TAG), \%. All analyses were done in duplicate. The adopted values are the means at the $95 \%$ confidence limit.

\section{Fatty Acids Methyl Ester Analysis by GC}

The FA compositions of fractions were investigated by gas chromatography (Thermo Trace GC Ultra, USA) equipped with an autosampler, a flame ionization detector and a Omegawax 250 fused silica capillary column (30 m x $0.25 \mathrm{~mm} \times 0.25 \mu \mathrm{m}$ film thickness; Supelco, Bellefonte, PA, USA). Helium was used as the carrier gas with a flow rate of $1 \mathrm{~mL} / \mathrm{min}$. A temperature program was set as follows: increasing from $170^{\circ} \mathrm{C}$ to $215^{\circ} \mathrm{C}$ at a rate of $1{ }^{\circ} \mathrm{C} / \mathrm{min}$, held at

Table 1. Reaction Conditions Employed to Investigate their Effects on Omega 3 PUFA Concentration by CR Lipase-Catalyzed Hydrolysis ${ }^{\mathrm{a}}$

\begin{tabular}{|c|c|c|c|}
\hline Reaction no & Buffer-to-oil ratio, v/w (mL:g) & Surfactant $^{\mathbf{b}}$ & Enzyme load, $^{\mathbf{c}}$ \\
\hline \hline I & $4: 1$ & - & 0.01 \\
\hline II & $4: 1$ & + & 0.01 \\
\hline III & $2: 1$ & + & 0.01 \\
\hline IV & $2: 1$ & + & 0.01 \\
\hline V & $2: 1$ & + & 0.05 \\
\hline VI & $2: 1$ & + & 0.1 \\
\hline
\end{tabular}

${ }^{\mathrm{a}}$ All reactions were performed with $300 \mathrm{rpm}$ of stirring for $8 \mathrm{~h}$. Sonication step was omitted.

${ }^{\mathrm{b}}$ Oil amount was kept constant at $3 \mathrm{~g}$.

c" +" indicates that Tween 80 at the amount of $1 \%(\mathrm{w} / \mathrm{v}$, based on buffer volume) was added. 
$215^{\circ} \mathrm{C}$ for $15 \mathrm{~min}$. The injector and detector temperatures were set at $250^{\circ} \mathrm{C}$ and $270^{\circ} \mathrm{C}$, respectively. FAs were identified by comparing their retention times with standard mixtures and expressed as wt $\%$ after correction for detector response factors.

\section{RESULTS AND DISCUSSION}

The FA profile of the salmon oil is shown in Table 2. The dominant FA in the substrate was oleic acid (OA, 18:1) accounting for $35.51 \%$ of total FAs, followed by palmitic acid (16:0) with a content of $12.19 \%$. MUFA fraction had the highest portion (48.2\%), which was believed to be resulted from the feed given in the salmon farm. Total omega 3 PUFA content, consisted of EPA, docosapentaenoic acid (DPA) and DHA, was $13.77 \%$.

Table 2. Fatty Acid Composition of Salmon Oil

\begin{tabular}{|c|c|}
\hline Fatty acid & Content, wt \% \\
\hline $14: 0$ & 3.78 \\
\hline $16: 0$ & 12.19 \\
\hline 18:0 & 2.39 \\
\hline $20: 0$ & 0.22 \\
\hline$\Sigma$ SFA & 18.58 \\
\hline $16: 1$ & 4.72 \\
\hline $18: 1 \mathrm{n}-7$ & 7 \\
\hline $18: 1 \mathrm{n}-9$ & 28.51 \\
\hline $20: 1$ & 4.77 \\
\hline $22: 1$ & 3.2 \\
\hline$\Sigma$ MUFA & 48.2 \\
\hline $18: 2$ & 10.64 \\
\hline $18: 3$ & 3.07 \\
\hline $18: 4$ & 0.83 \\
\hline $20: 2$ & 0.33 \\
\hline $20: 3 n-3$ & 0.36 \\
\hline $20: 3 n-6$ & 0.23 \\
\hline $20: 4 n-3$ & 1.03 \\
\hline $20: 4 n-6$ & 0.42 \\
\hline 20:5 n-3 EPA & 4.8 \\
\hline $22: 5 n-3$ DPA & 2.04 \\
\hline 22:6 n-3 DHA & 6.93 \\
\hline इPUFA & 30.68 \\
\hline EPA + DPA + DHA & 13.77 \\
\hline
\end{tabular}

\section{Screening of Lipases}

The aim of this work was to select the best lipase in terms of concentrating omega 3 PUFA in the glyceride frac- tion by hydrolysis. Theoretical upgrading steps are illustrated in Fig. (1). The candidate lipase should have the substrate specificity towards SFA and MUFA in order to release these FAs while recovering omega 3 PUFA in the glyceride fraction.

\section{Evaluation Based on Hydrolysis Degree}

The first step was to monitor the hydrolysis efficiency of the lipases used. Fig. (2) shows the time courses of hydrolysis reactions performed by different lipases. The hydrolysis reaction reached equilibrium for all the lipases tested after 12 to $24 \mathrm{~h}$. The lipases can be roughly divided into two groups according to their hydrolysis rate and HD. Lipases originating from PC, RJ, and RN had low hydrolysis rates and were grouped together. After the first $30 \mathrm{~min}$, the HD values obtained by these lipases were $11.97 \%, 19.68 \%$, and $9.42 \%$, respectively. In the second group of lipases, on the other hand, both hydrolysis rate and HD were significantly higher. HD reached to $35.33 \%, 47.24 \%, 47.33 \%$, and $54.97 \%$, respectively, by the lipases from CR, RM, BC, and RO after the first $30 \mathrm{~min}$. Although the HD value after $30 \mathrm{~min}$ was much lower (21.71\%) for the RD lipase compared to the rest of the group, the conversion of TAG was almost complete after $24 \mathrm{~h}(91.72 \%)$, and thus, this lipase was placed in the second group as well.

Hydrolysis efficiencies of lipases depend on many conditions, such as the emulsification of the phases, temperature and $\mathrm{pH}$ of the reaction medium, ratio between the oil and the aqueous phase, reaction period, etc. For instance, HD value obtained by CR lipase (91.89\%), which has no positional specificity, was equal to that obtained by RD lipase $(91.72 \%)$, which hydrolyzes $s n-1,3$ positions specifically $[5$, 10]. Besides, no differences between immobilized and free lipases were observed in terms of hydrolysis efficiency. RM, the only immobilized lipase used in the study, had a moderate HD level. From an industrial point of view, immobilized form is preferred due to the repeated use of the enzyme. However, the compensation by the increased reaction rate was considered to be a solid reason to investigate the free lipases further. Within the reaction conditions employed in the present study, the lipases in the second group, namely the ones originating from $\mathrm{CR}, \mathrm{RM}, \mathrm{BC}, \mathrm{RO}$, and $\mathrm{RD}$, had been chosen to conduct the further steps of the study.

\section{Evaluation Based on Omega 3 PUFA Content in Glyc- erides}

Table 3 shows the FA content in the glyceride fraction after $24 \mathrm{~h}$ of hydrolysis catalyzed by selected lipases. RM and RD lipases increased the omega 3 PUFA content at the initial stage of the reaction, but the trend was reversed after 1 $\mathrm{h}$ (data not shown). The final omega 3 PUFA content observed was $10.19 \%$ by RM and $8.89 \%$ by RD after $24 \mathrm{~h}$ reaction. Compared to the initial omega 3 PUFA content of salmon oil (13.77\%), these lipases caused the loss of the target FAs, and thus, were not considered further. Once again, no advantage of immobilization of RM lipase was observed for the purpose of the study. Nwosu and Byond [16] reported that the FAs were distributed randomly among the TG molecules found in salmon oil. The majority of OA, which was the FA with the highest content in the salmon oil used, and therefore, the target FA to be removed from the desired product, was located at the $s n-1,3$ positions; however, more 


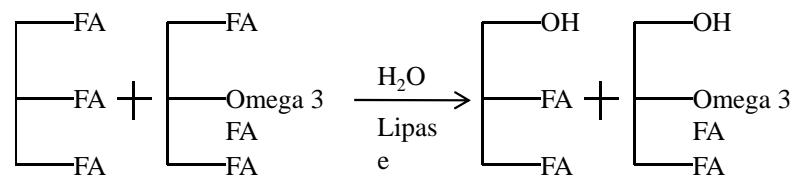

16\% Omega 3 FA

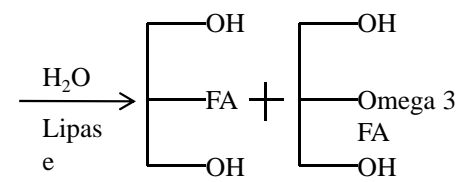

50\% Omega 3 FA

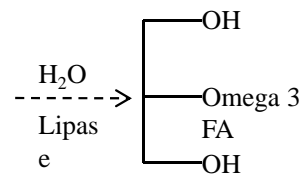

100\% Omega 3 FA

Fig. (1). Schematic illustration of enzymatic upgrading of farmed salmon oil catalyzed by a lipase selective for SFA and MUFA. Hydrolysis reaction results in releasing of SFA and MUFA to the FFA fraction, while recovering target omega 3 PUFA in the glyceride fraction, thereby, increasing the value of the salmon oil. SFA-saturated fatty acids, MUFA-monounsaturated fatty acids, FFA-free fatty acids, PUFApolyunsaturated fatty acids.

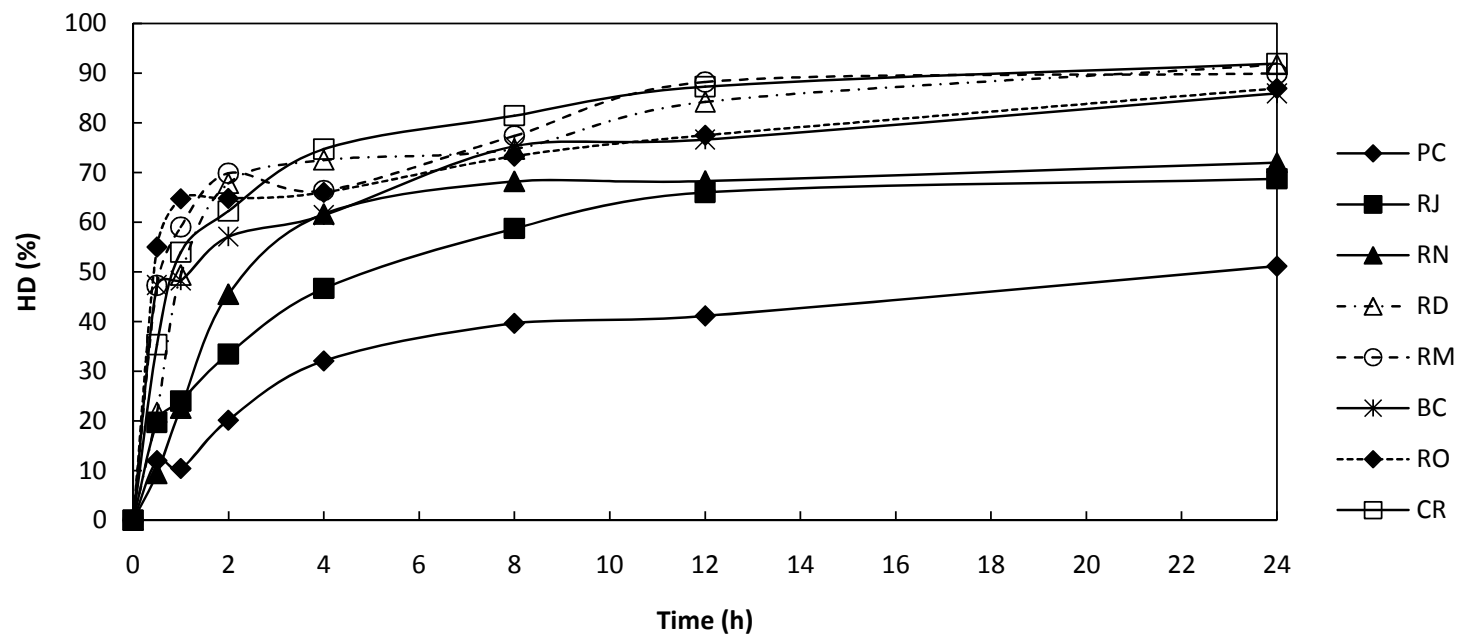

Fig. (2). Time courses of hydrolysis reactions catalyzed by different lipases. Reactions were performed with $3 \mathrm{~g}$ oil and $6 \mathrm{~mL}$ buffer, and catalyzed by $40 \mathrm{U} / \mathrm{g}$ oil lipase at $37^{\circ} \mathrm{C}$ under $700 \mathrm{rpm}$ agitation for $24 \mathrm{~h}$.

than half of EPA, DPA and DHA were identified at these positions as well. Both RM and RD lipases are $s n-1,3$ specific, which can be the reason for the decrease in omega 3 PUFA content. Similar conclusion has been obtained previously as well $[3,11]$.

$\mathrm{RO}$ and $\mathrm{BC}$ lipases resulted in a minor improvement in omega 3 PUFA content by increasing the value from $13.77 \%$ to $17.88 \%$ and $14.52 \%$, respectively. However, considering the long reaction time, the improvement was inadequate. CR lipase, on the other hand, resulted in a tremendous increase in omega 3 PUFA content. The value was increased by approximately $50 \%(20.54 \%)$ after $2 \mathrm{~h}$ and reached to $27.81 \%$ at the end of $24 \mathrm{~h}$ period. CR lipase is one of the most widely used lipases for the enzymatic production of omega 3 PUFA concentrates due to its high hydrolysis efficiency and discrimination against EPA and DHA [3-6, 9-12, 17]. This lipase had no positional specificity, hydrolyzing FAs at all snpositions randomly even at HD levels as low as $20 \%$. On the other hand, it had strict acyl chain specificity, resulting in selective hydrolysis of SFA and MUFA at a much higher rate compared to omega 3 PUFA [18].

\section{Fatty Acid Selectivity of Lipases}

Fig. (3) shows the changes in total omega 3 PUFA content related to HD during hydrolysis catalyzed by the selected lipases. The changes in omega 3 PUFA content were significantly different although the HD values were similar for all the lipases at the end of $24 \mathrm{~h}$. CR lipase resulted in

Table 3. FA Content in the Glyceride Fraction After $24 \mathrm{~h}$ of Hydrolysis Catalyzed by Selected Lipases

\begin{tabular}{|c|c|c|c|c|c|}
\hline Origin of lipase & \multicolumn{5}{|c|}{ FA content, wt $\%$} \\
\hline $\mathrm{RD}$ & 42.2 & 2.07 & 1.58 & 5.24 & 8.89 \\
\hline RO & 26.83 & 4.38 & 2.37 & 11.14 & 17.88 \\
\hline $\mathrm{CR}$ & 20.91 & 5.9 & 2.52 & 19.39 & 27.81 \\
\hline
\end{tabular}




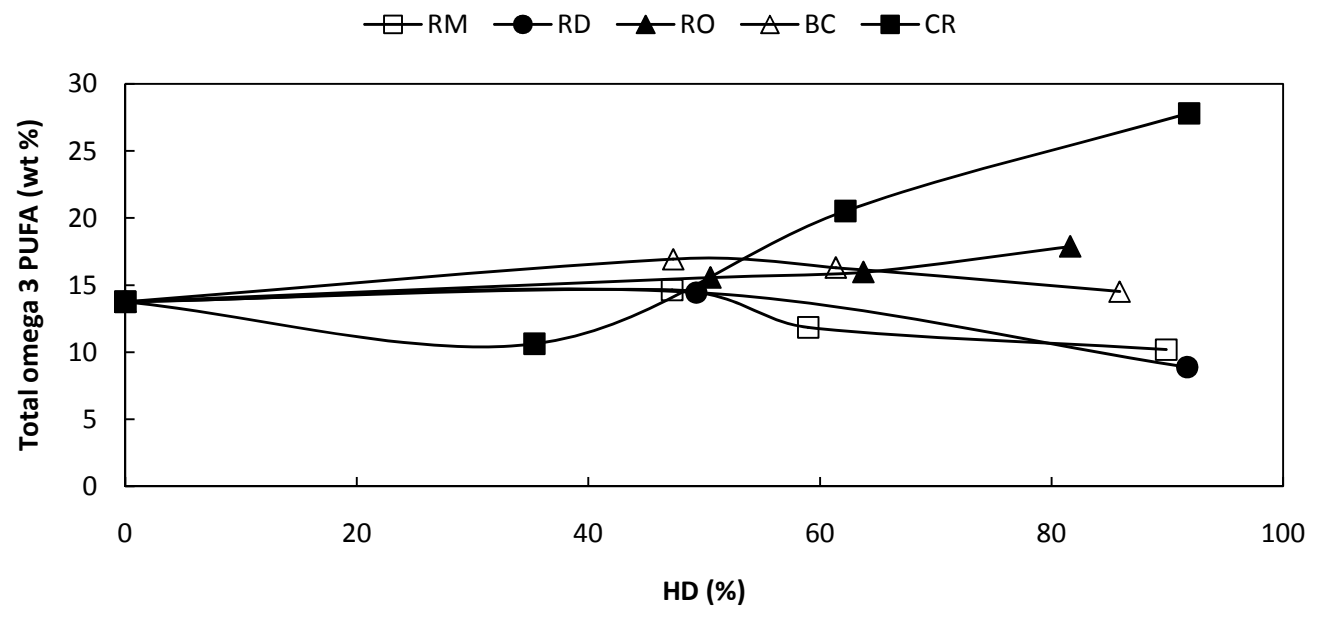

Fig. (3). Changes in total omega 3 PUFA content related to hydrolysis degree (HD) during hydrolysis catalyzed by selected lipases. See Fig. (2) for reaction conditions.

constant increase in total omega 3 PUFA content with increasing HD above $40 \%$, while the rest of the lipases caused a slight decrease above $50 \%$ of HD. This trend was believed to be caused by the substrate specificities of the lipases.

In heterogeneous reactions, such as hydrolysis of oil, the reaction rate is determined by the emulsion conditions, affected by many parameters including buffer-to-oil ratio, stirring rate, shape of the reactor used, and the presence of surfactants. The reaction time depends on these factors, and therefore, it is difficult to adequately estimate the specificities of lipases. However, Tanaka et al. [6] suggested that the relationship between the hydrolysis resistant value (HRV) and HD discounted the reaction time factor. HRV was calculated as follows:

$H R V(\%)=\left\{\left(100 \times G C_{a}-B \times G C_{b}\right) \div\left(100 \times G C_{a}\right)\right\} \times 100$

where $G C_{a}$ is the content of each FA in the original oil and $G C_{b}$ is the content of each FA in the FFA fraction of the product, both of which were measured by GC (wt \%), and $B$ is the ratio of FFA in the hydrolyzed oil, measured by TLCFID (vol \%). A high HRV indicates a high resistance of the FA of interest to hydrolysis.

HRV values for EPA, DHA, and OA were calculated for the hydrolysis reactions catalyzed by selected lipases. Fig. (4) shows the relationship between HRV and HD for EPA, DHA, and OA. All of the lipases used had a high resistance towards hydrolysis of EPA at the initial stages of the reactions (Fig. 4a). Starting from HD of approximately $40 \%$, HRV decreased steadily until the end of the $24 \mathrm{~h}$ period. The most significant decrease was observed for RD lipase, which had a negative HRV above $80 \%$ hydrolysis, indicating that the concentration of EPA was higher in the FFA fraction compared to the glyceride fraction. HRV calculated for EPA was the highest for CR lipase until HD of $60 \%$ and decreased with increased hydrolysis. A similar trend was observed for the resistance of DHA against hydrolysis (Fig. 4b).

Apart from RD lipase, all lipases seemed to have high discrimination against hydrolysis of EPA and DHA. Thus, the differences in the relative total omega 3 PUFA content must have resulted from the overall composition of the reaction products. Fig. (3c) shows the relationship between HD and HRV for OA. The HRV pattern for OA was significantly different compared to those for EPA and DHA. CR lipase had the lowest value throughout the reaction, while the rest of the lipases had a similar trend until HD of approximately $40 \%$, after which HRV had a sharp decrease.

The investigation of HRV for different lipases cleared the reason for varying concentration of omega 3 PUFA by hydrolysis, despite the similar HD reached by all of the lipases used. Considering HRV in relation to HD for EPA, DHA and $\mathrm{OA}$, it can be concluded that the concentration of omega 3 PUFA in salmon oil depends on the discrimination of the lipase against EPA and DHA, as well as the selective hydrolysis of OA, the target FA.

CR lipase was reported to recognize not only the FAs at the positions of TG separately, but also the whole TG molecule $[6,19]$. It was suggested that hydrolysis reaction catalyzed by CR lipase took place in two steps: TG molecules without DHA were hydrolyzed at the earlier stages of the reaction. As the reaction progressed, DHA containing-TG molecules were hydrolyzed as well. This was believed to be the reason for the decreases in HRV calculated for EPA and DHA concerning the later stages of CR lipase-catalyzed hydrolysis.

In order to justify the reliability of the approach, HRV calculations were made for the set of experiments with different conditions which were performed by CR lipase to see the effects of reaction conditions. Although the obtained FA profiles were quite different among the reactions (Fig. 5), the HRV patterns for EPA, DHA and OA were not affected by the presence of surfactants, by eliminating the sonication step, by increasing the buffer-to-oil ratio, or by increasing the enzyme load (data not shown). Hence, HRV pattern is a useful tool to determine the FA selectivity of lipases.

\section{Effects of Reaction Conditions on CR Lipase-Catalyzed Hydrolysis of Salmon Oil}

Since the screening step concluded that lipase from CR resulted in the highest total omega 3 PUFA content, a series 
a

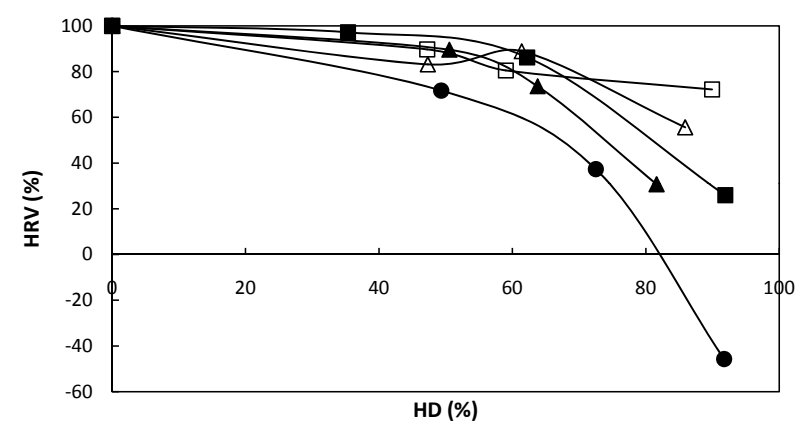

b
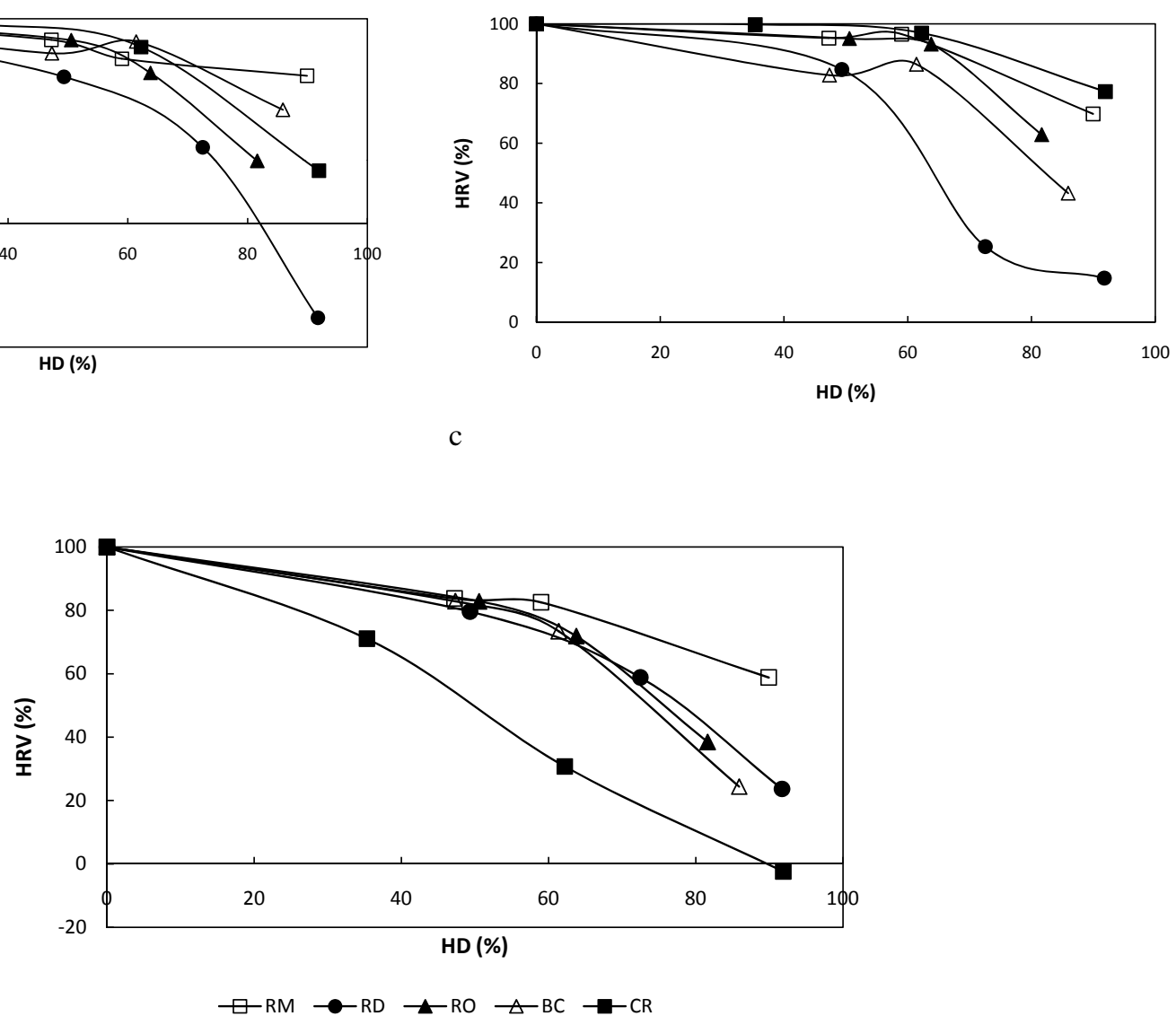

Fig. (4). Relationship between hydrolysis degree (HD) and hydrolysis resistant value (HRV) for (a) EPA; (b) DHA; (b) OA. See Fig. (2) for reaction conditions. EPA-eicosapentaenoic acid, DHA-docosahexaenoic acid, OA-oleic acid.

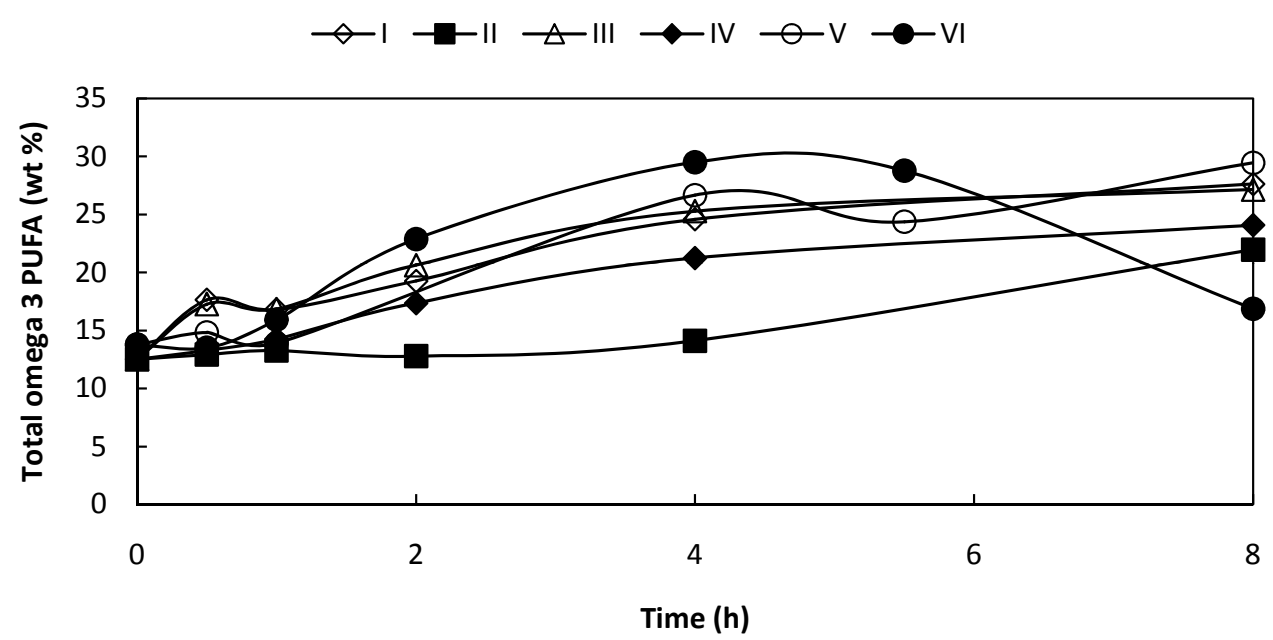

Fig. (5). Changes in total omega 3 PUFA content of the glyceride fraction during hydrolysis. See Table 1 for reaction conditions.

of reactions with varying conditions were performed to determine the effects of the parameters on the reaction, as well as to confirm the HRV pattern was not influenced by the reaction conditions. Fig. (5) represents the PUFA concentra- tion pattern obtained by different conditions. Fig. (6) shows the time course of the hydrolysis reactions. The effects of the conditions are discussed below separately. 


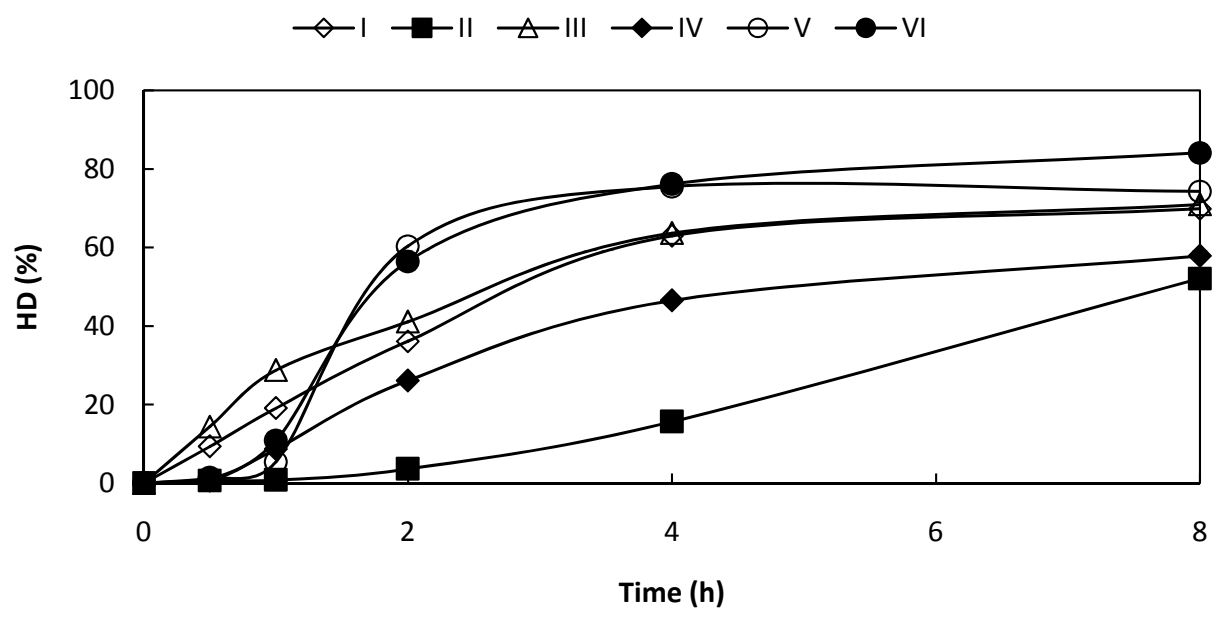

Fig. (6). Time courses of hydrolysis reactions. See Table $\mathbf{1}$ for reaction conditions.

\section{Effect of Emulsification}

Reactions I and II had identical conditions except that reaction I had no surfactant added while reaction II included $1 \%$ of surfactant in the buffer phase (w/v). Hydrolysis rate was much slower in reaction II compared to that in reaction I (Fig. 6), presumably because it was more difficult for the enzyme to act on the emulsified substrate compared to the unbound oil phase. Moreover, emulsification resulted in decreased concentration of PUFA (Fig. 5), linked to low HD. Reactions III and IV were another set of experiments with identical conditions other than the use of surfactant. The observed trend for HD and omega 3 PUFA concentration were similar to those obtained by the comparison of reactions I and II. Lipases are enzymes which function at the interface of the heterogeneous system, and the use of surface active agents that form microemulsions, or the use of a reverse micellar system, is expected to enhance lipase activity [20]. Tween 80 has been reported to enhance the activity and enantioselectivity of CR lipase [21]. The lipase was activated since Tween 80 is non-ionic, so there were only hydrogenbonding and hydrophobic interactions with the lipase. On the other hand, the study also reported that the enhancing effect was highly dependent on the concentration of the surfactant. The added surfactant in the present study was at the level of $1 \%$ (w/v), which could be the reason for the low enzymatic activity. Salgin and Takac [22] investigated the effect of another non-ionic surfactant, Triton X-100, on the activity of CR lipase in a biphasic medium composed of phosphate buffer solution and isooctane. Added surfactant at concentrations in the range of 0.2 to $0.5 \mathrm{mM}$ stimulated the hydrolytic activity, in contrary to the present results. The contradictory conclusion is believed to be a result of the different procedure followed. In the cited study, the lipase was incubated in the biphasic medium at the presence of surfactant for $24 \mathrm{~h}$ prior to the reaction, which was claimed to lead to a conformational change in the protein structure in a way to improve the activity at the water-isooctane interfacial area [22]. Byun et al. [20] compared HD obtained by porcine pancreas lipase with and without emulsification by gelatin. In the case that gelatin was added to the aqueous phase, which was water in their system, HD value was 4-fold of that obtained by the non-emulsified reaction after $48 \mathrm{~h}$. The highly contradictory conclusion could have been a result of the prolonged reaction time. Considering the cost issues, short reaction time is preferred. All in all, the use of surfactant had negative effect on both HD and total omega 3 PUFA content, and aqueous system without added emulsifier was considered to be more preferable for the hydrolysis reaction.

\section{Effect of Buffer-to-Oil Ratio}

Reactions I vs. III and II vs. IV were compared to see the effect of buffer-to-oil ratio on HD and total omega 3 PUFA content. HD value increased slightly when the amount of buffer was doubled (Fig. 6), which was believed to be a result of the increased interphase area that was available for hydrolytic activity. It has been reported that, even after a 10fold increase in the ratio between the aqueous and the oil phases, the increase in HD was only by $8 \%$ [23]. On the other hand, the increase in the buffer amount did not have a significant effect on the total omega 3 PUFA content (Fig. 5). In fact, the DHA content was slightly lower in reaction IV compared to that in reaction II (data not shown). Considering both parameters, it had been concluded that 2-fold buffer (based on oil amount) was more preferable over 4fold.

\section{Effect of Enzyme Load}

Reactions IV, V and VI were performed by varying amounts of lipase with other reaction conditions being constant. HD was increased when enzyme load was higher (Fig. 6), especially when it was increased by 5 -fold (from reaction IV to reaction V). This result was obviously expected [11, 23-26], since the rate limiting component in the enzymatic reactions with high concentrations of substrates is the amount of enzyme. The trend observed in the total omega 3 PUFA content was somewhat different (Fig. 5). The amount of omega 3 PUFA obtained by the reactions IV and V were about double of that found in the substrate oil. Reaction VI, on the other hand, reached the same level at $4 \mathrm{~h}$ instead of 8 , after which the omega 3 PUFA content decreased back to the original value. Enzyme load in reaction VI was 10 times higher than that in reaction IV, and 2-fold of the amount in 
reaction $\mathrm{V}$. The reason for the omega 3 PUFA content to decrease in the later stages of the reaction VI was assumed to be the fact that, as mentioned before, the HD level of the reactions with high enzyme load was higher. Therefore, CR lipase started to act on the omega 3 PUFA ester bonds as well, and resulted in their loss to the FFA fraction [6, 19, 25]. Gamez-Meza et al. [25] observed a similar trend, but with much higher enzyme load. Omega 3 PUFA content decreased when the enzyme load was increased by 10 -fold, while 2-fold increase was sufficient to see the same effect in another study [11]. Considering the cost issues, fast and efficient concentration of omega 3 PUFA is the primary goal, therefore, $0.1 \mathrm{~g}$ of enzyme load was considered to be the best choice.

\section{CONCLUSION}

The present study showed that lipase-catalyzed hydrolysis resulted in successful concentration of omega 3 PUFA in salmon oil obtained from by-products of salmon production. Despite similar HD values reached by different lipases, the changes in omega 3 PUFA content were significantly different, which was believed to be a result of their substrate specificities. Lipase from CR was shown to be the most effective one considering both HD and omega 3 PUFA content levels. Investigation of HRV revealed that the efficiency of a lipase to concentrate omega 3 PUFA depended on its discrimination against EPA and DHA, as well as selective removal of SFA and MUFA. HRV was not affected by varying reaction conditions, providing a reliable parameter to judge the FA selectivity of lipases. Further investigation of the effects of the reaction conditions on CR lipase-catalyzed hydrolysis showed that it is possible to obtain as high omega 3 PUFA levels as 2.15-fold of the original content.

\section{PRACTICAL APPLICATIONS}

For farmed fish, the omega 3 content is usually reduced due to feed containing mainly vegetable oils where a limited amount of long chain PUFA is available. The fish oil value is, therefore, reduced for food uses. This brings huge concern for the fish farming industry, despite the fact that the fish oil quality is considered to be high for food uses due to controlled pollution and better processing operations. Improvement of the omega 3 PUFA content to a high level, at least no less than wild fish oils, is highly demanded, especially by simple, mild and cost efficient processing. In this work, we aimed to develop a simple process for the upgrading of the farmed fish oil for food uses. The content of omega 3 PUFA is easily doubled by the processing.

\section{ACKNOWLEDGEMENT}

The authors thank Aarhus University and Marine Bioproducts A/S (Bergen, Norway) for the financial support.

\section{ABBREVIATIONS}

$\begin{array}{ll}B C & =\text { Burkholderia cepacia } \\ C R & =\text { Candida rugosa } \\ \text { DAG } & \text { diacylglycerols } \\ \text { DHA } & =\text { docosahexaenoic acid } \\ \text { DPA } & \text { docosapentaenoic acid } \\ \text { EPA } & =\text { eicosapentaenoic acid }\end{array}$

$\begin{array}{ll}\mathrm{FA} & =\text { fatty acid } \\ \mathrm{FFA} & =\text { free fatty acids } \\ \mathrm{HD} & =\text { hydrolysis degree } \\ \mathrm{HRV} & =\text { hydrolysis resistant value } \\ \mathrm{MAG} & =\text { monoacylglycerols } \\ \mathrm{MUFA} & =\text { monounsaturated fatty acids } \\ \mathrm{OA} & =\text { oleic acid } \\ \text { PC } & =\text { Penicillium camembertii } \\ \mathrm{PUFA} & =\text { polyunsaturated fatty acids } \\ R D & =\text { Rhizopus delemar } \\ R J & =\text { Rhizomucor javanicus } \\ R M & =\text { Rhizomucor miehei } \\ R N & =\text { Rhizopus niveus } \\ R O & =\text { Rhizopus oryzae } \\ \mathrm{SFA} & =\text { saturated fatty acids } \\ \mathrm{TAG} & =\text { triacylglycerols }\end{array}$

\section{REFERENCES}

[1] Yashodhara BM, Umakanth S, Pappachan JM, Bhat SK, Kamath R, Choo BH. Omega-3 fatty acids: a comprehensive review of their role in health and disease. Postgrad Med J 2009; 85: 84-90.

[2] Shahidi F, Wanasundara UN. Omega-3 fatty acid concentrates: Nutritional aspects and production technologies. Trends Food Sci Technol 1998; 9: 230-40.

[3] Sun T, Pigott GM, Herwig RP. Lipase-assisted concentration of n-3 polyunsaturated fatty acids from viscera of farmed Atlantic salmon (Salmo salar L.). J Food Sci 2002; 67: 130-6.

[4] Lie O, Lambertsen G. Fatty acid specificity of Candida cylindracea lipase. Fette Seifen Anstrichmittel 1986; 88: 365-7.

[5] Hoshino T, Yamane T, Shimizu S. Bioreactor for enzymaticreaction of fat and fatty-acid derivatives. 12. Selective hydrolysis of fish oil by lipase to concentrate n-3 polyunsaturated fatty-acids. Agric Biol Chem 1990; 54: 1459-67.

[6] Tanaka Y, Hirano J, Funada T. Concentration of docosahexaenoic acid in glyceride by hydrolysis of fish oil with Candida cylindracea lipase. J Am Oil Chem Soc 1992; 69: 1210-4.

[7] Mukherjee KD, Kiewitt I, Hills MJ. Substrate specificities of lipases in view of kinetic resolution of unsaturated fatty acids. Appl Microbiol Biotechnol 1993; 40: 489-93.

[8] Shimada Y, Sugihara A, Maruyama K, et al. Enrichment of arachidonic acid - selective hydrolysis of a single-cell oil from Mortierella with Candida cylindracea lipase. J Am Oil Chem Soc 1995; 72: 1323-7.

[9] Moore SR, McNeill GP. Production of triglycerides enriched in long-chain n-3 polyunsaturated fatty acids from fish oil. J Am Oil Chem Soc 1996; 73: 1409-14.

[10] Wanasundara UN, Shahidi F. Lipase-assisted concentration of n-3 polyunsaturated fatty acids in acylglycerols from marine oils. J Am Oil Chem Soc 1998; 75: 945-51.

[11] Okada T, Morrissey MT. Production of $n-3$ polyunsaturated fatty acid concentrate from sardine oil by lipase-catalyzed hydrolysis. Food Chem 2007; 103: 1411-9.

[12] Lyberg AM, Adlercreutz P. Lipase specificity towards eicosapentaenoic acid and docosahexaenoic acid depends on substrate structure. Biochim Biophys Acta: Proteins Proteomics 2008; 1784: 34350 .

[13] Sorensen S, Sandnes K, Hagen H, Pedersen K, inventors; Apparatus and method for hydrolysis of a protein containing raw material and application of the resulting hydrolysis products. European Patent EP 1575374 B1. 2004.

[14] Japanese Industrial Standards Committee. Japanese Industrial Standard, JIS K601. 1988.

[15] American Oil Chemists' Society (AOCS). AOCS method Ce-1b 89 2007. 
[16] Nwosu CV, Boyd LC. Positional distribution of fatty acids on triacylglycerols of menhaden (Brevoortia tyrannis) and salmon (Salmo salar) oils. J Food Lipids 1997; 4: 65-74.

[17] Halldorsson A, Kristinsson B, Haraldsson GG. Lipase selectivity toward fatty acids commonly found in fish oil. Eur J Lipid Sci Technol 2004; 106: 79-87.

[18] Benzonana G, Esposito S. Positional and chain specificities of Candida cylindracea lipase. Biochim Biophys Acta 1971; 231: 1522.

[19] Tanaka Y, Funada T, Hirano J, Hashizume R. Triglyceride specificity of Candida cylindracea lipase - effect of docosahexaenoic acid on resistance of triglyceride to lipase. J Am Oil Chem Soc 1993; 70: 1031-4.

[20] Byun HG, Eom TK, Jung WK, Kim SK. Lipase-catalyzed hydrolysis of fish oil in an optimum emulsion system. Biotechnol Bioprocess Eng 2007; 12: 484-90.

[21] Liu YY, Xu JH, Hu Y. Enhancing effect of Tween-80 on lipase performance in enantioselective hydrolysis of ketoprofen ester. $\mathbf{J}$ Mol Catal B: Enzym 2000; 10: 523-9.
[22] Salgin S, Takac S. Effects of additives on the activity and enantioselectivity of Candida rugosa lipase a in a biphasic medium. Chem Eng Technol 2007; 30: 1739-43.

[23] Shimada Y, Maruyama K, Okazaki S, Nakamura M, Sugihara A, Tominaga Y. Enrichment of polyunsaturated fatty-acids with Geotrichum candidum lipase. J Am Oil Chem Soc 1994; 71: 951-4.

[24] Shimada Y, Maruyama K, Sugihara A, Moriyama S, Tominaga Y. Purification of docosahexaenoic acid from tuna oil by a two-step enzymatic method: Hydrolysis and selective esterification. J Am Oil Chem Soc 1997; 74: 1441-6.

[25] Gamez-Meza N, Noriega-Rodriguez JA, Medina-Juarez LA, et al. Concentration of eicosapentaenoic acid and docosahexaenoic acid from fish oil by hydrolysis and urea complexation. Food Res Int 2003; 36: 721-7.

[26] Kojima Y, Sakuradani E, Shimizu S. Different specificity of two types of Pseudomonas lipases for $\mathrm{C} 20$ fatty acids with a $\Delta 5$ unsaturated double bond and their application for selective concentration of fatty acids. J Biosci Bioeng 2006; 101: 496-500.

(c) Kahveci et al:; Licensee Bentham Open.

This is an open access article licensed under the terms of the Creative Commons Attribution Non-Commercial License (http://creativecommons.org/licenses/by-nc/3.0/) which permits unrestricted, non-commercial use, distribution and reproduction in any medium, provided the work is properly cited. 